\title{
Unmanned aerial vehicle for post seismic and other hazard scenarios
}

\author{
V. Baiocchi ${ }^{1}$, D. Dominici ${ }^{2} \&$ M. Mormile ${ }^{1}$ \\ ${ }^{I}$ DICEA - Area Geodesia e Geomatica, "Sapienza", \\ University of Rome, Italy \\ ${ }^{2}$ Department of Architecture and City Planning, \\ University of L'Aquila, Italy
}

\begin{abstract}
On April 6, 2009, an earthquake hits the old city of L'Aquila resulting in hundreds of victims and thousands of collapses. The post emergency and very crucial phase regarding surveys, structure controls and investigations till now is fully underway. Conventional surveying techniques using high precision total stations, GNSS and laser scanners, are instruments of extreme operability but there are still many evident limits on their use especially regarding the survey of both the roofs and the facades of tall buildings or dangerous places, typical in post earthquake situations. Another very important disadvantage of the traditional surveying, especially during the post seismic periods, regards the overall safety of the instruments, operators and workers. In particular, the seismic sequence that may last for months after the main shock, causing collapses and ulterior damage that in most cases can also damage the transport and communication infrastructure creating important problems for all instruments located in that area and definitely posing a further serious risk for all operators. Moreover, some surveys have to be repeated periodically in order to achieve a final result. On the other hand, by using micro UAVs (Unmanned Aerial Vehicles) for surveying in such particular scenarios, many of these problems can easily bypassed. For example, there are no accessibility problems for an UAV mainly because of its extreme flying capabilities that can really permit the access of virtually any place. Regarding the security, these instruments are fully remotely controlled, so they do not involve any risk for the operators simply because they are in the dangerous area. For these reasons, in this work, we'll explain the advantages of multirotor UAVs, fully remotely
\end{abstract}


controlled, to acquire roofs and facades of structures in an old city center damaged by a seismic sequence like L'Aquila city.

Keywords: UAV, earthquake, flight planning, L'Aquila, UP23d.

\section{Introduction}

The development of electronics, the camera performance improvement, and their ability to transmit static and dynamic images, led to the introduction of a new technique of survey: photogrammetry with UAV (Unmanned Aerial Vehicle). UAVs are aircrafts remotely piloted from a ground station, and represent the last frontier for the acquisition of the territory with high resolution and low-altitude flight. Their ability to carry different sensors makes them usable in urban areas, for the analysis of the risk environment and for the estimation of the changes of land use, for example. They also represent a low-cost solution for the creation of shared cognitive frameworks. In particular, in the field of Civil Protection, where the absolute priority is to preserve life, these platforms are used for the observation and data collection in areas affected by earthquakes, landslides, subsidence, avalanches, or for the control of forests and the prevention of summer fires.

Public Administrations are increasing the studies of drones to carry out investigations in the territory and, in particular, to perform photogrammetric proximity surveys, to detect areas of small extensions, in short times and with reduced costs very low. This scope is the subject of constant scientific research, as the technical requirements of shooting for photogrammetric purposes requiring high precision of the instruments used, and certain conditions for the taking for images to be captured. This application was selected and tested in this work for a documentation of the damage caused in the historic center of L'Aquila earthquake of April 2009.

\section{The UAV technology}

Born in the military field for reconnaissance and intelligence operations, UAVs are in continuous development for civil applications, particularly in the role of measuring instruments to survey the area. Thanks to new developed navigation systems that ensures their reliability and stability, it's now possible acquire actual photogrammetric imagery from UAV. An increasing variety of equipment is presently tested from the scientific community to be installed on board as video/digital cameras, thermal cameras Lidar, atmospheric sensors or other types of sensors.

Great research work in recent years has allowed the developing of different types of UAVs; it should therefore make a preliminary classification, on the basis of the properties that best distinguish them:

In table 2, the existing classes of UAV are shown, considering the parameters described above:

Among these, the HALE platforms are larger in size and are used daily in surveillance and reconnaissance missions. The flight altitude for these vehicles is 
Table 1: $\quad$ Properties of different types of UAVs.

\begin{tabular}{|c|c|}
\hline Dimensions & Description \\
\hline Operational flight altitude (m) & $\begin{array}{c}\text { maximum flight altitude within is } \\
\text { guaranteed proper operation }\end{array}$ \\
\hline Operating time (hours) & $\begin{array}{c}\text { function of the size of the aircraft, the } \\
\text { type of power and especially of the } \\
\text { transported load }\end{array}$ \\
\hline Payload (kg) & $\begin{array}{c}\text { transportable weight recommended } \\
\text { in order to ensure good battery life }\end{array}$ \\
\hline Action radius & \begin{tabular}{c} 
aircraft can operate \\
extension of the surface on which the \\
\hline
\end{tabular} \\
\hline
\end{tabular}

always kept high in order not to obstruct the field of action of the UAV MALE, which operate at lower altitudes and which have the purpose (in the military field) to support ground units providing in real time a series of high-resolution images. Very popular for civil applications are categories Mini, Micro and Nano UAV, operating at an altitude of no more than $300 \mathrm{~m}$ and within a maximum radius of $10 \mathrm{~km}$. These have dimensions and load capacity reduced compared to previous and are equipped with inertial sensors of type MEMS (Micro Electrical Mechanical Systems) at low cost, that can reduce the size and weight of these platforms while maintaining excellent stability of flight. We'll focus our investigation on the 'Multirotor' category type Micro, which is the most diffused in civil applications as technology 'low-cost'.

\section{The current legislation}

International organizations and bodies, although of various projects and programs, still didn't define clear standards, specifications and certifications commonly accepted for UAV platforms, so each country has their own guidelines to follow, to ensure the safety of population. Presently the regulation 
116 Safety and Security Engineering V

Table 2: $\quad$ Classification of existing UAV.

\begin{tabular}{|c|c|c|c|c|}
\hline Categories & Acronym & $\begin{array}{l}\text { Action } \\
\text { radius } \\
(\mathrm{Km})\end{array}$ & $\begin{array}{l}\text { Operational } \\
\text { flight altitude } \\
\text { (m) }\end{array}$ & $\begin{array}{c}\text { Operating } \\
\text { time } \\
\text { (hours) }\end{array}$ \\
\hline Nano & $\eta$ & $<1$ & 100 & $<1$ \\
\hline Micro & $\mu$ & $<10$ & 250 & $<1$ \\
\hline Mini & Mini & $<10$ & $150-300$ & $<2$ \\
\hline Close Range & $\mathrm{CR}$ & $10-30$ & 3000 & $2-4$ \\
\hline Short Range & SR & $30-70$ & 3000 & $3-6$ \\
\hline Medium Range & MR & $\begin{array}{l}70- \\
200\end{array}$ & 5000 & $6-10$ \\
\hline Categories & Acronym & $\begin{array}{l}\text { Action } \\
\text { radius } \\
(\mathbf{K m})\end{array}$ & $\begin{array}{l}\text { Operational } \\
\text { flight altitude } \\
\text { (m) }\end{array}$ & $\begin{array}{l}\text { Operating } \\
\text { time } \\
\text { (hours) }\end{array}$ \\
\hline $\begin{array}{l}\text { Medium Range } \\
\text { Endurance }\end{array}$ & MRE & $>500$ & 8000 & $10-18$ \\
\hline $\begin{array}{l}\text { Low Altitude Deep } \\
\text { Penetration }\end{array}$ & LADP & $>250$ & $50-9000$ & $0.5-1$ \\
\hline $\begin{array}{l}\text { Low Altitude Long } \\
\text { Endurance }\end{array}$ & LALE & $>500$ & 3000 & $>24$ \\
\hline $\begin{array}{l}\text { Medium Altitude Long } \\
\text { Endurance }\end{array}$ & MALE & $>500$ & 14000 & $24-48$ \\
\hline $\begin{array}{l}\text { High Altitude Long } \\
\text { Endurance }\end{array}$ & HALE & $>2000$ & 20000 & $24-48$ \\
\hline $\begin{array}{l}\text { Unmanned Combat Aerial } \\
\text { Vehicle }\end{array}$ & UCAV & $\begin{array}{c}\approx \\
1500\end{array}$ & 10000 & $\approx 2$ \\
\hline
\end{tabular}


of UAV systems in Italy is still not very clear, in particular for geomatic applications.

More in detail, for the Italian legislation, the use of aircraft over urban areas for the realization of cartography or urban analysis is presently regulated by the current ENAC (National Authority for Civil Aviation) legislation that prevents flights below $350 \mathrm{~m}$ above sea level. But this recommendation is obviously thought of for airplanes with a pilot on board so a statement from the FIAM (Italian Federation of Aero Modelling) states that UAVs, since they assume an automatic flight control, are not to be considered airplanes, because they are unmanned. So, it's to be evaluated which of these rules apply and how for every different type of UAV to ensure operational safety. The micro UAV systems that are mainly used in the field of geomatics usually fly at low altitudes (50-150 m).

\section{The UAV photogrammetry}

A correct photogrammetric survey needs a suitable number of high quality acquisitions with the correct geometry [1]. To assure this quality the whole flight and thus the acquisition points have to be spatially distributed maintaining definite geometric characteristics as equal and correct air base and correct interaxes [2]. Considering as a guideline, the case of a traditional aerophotogrammetric acquisition, a flight is planned according to predetermined almost rectilinear paths parallel to each other; during the flight, images are taken in succession at regular time intervals, forming what is called a 'swath'. To determine the density and the number of the needed waypoints some metric information are necessary. Firstly, the flying altitude and the flying speed to simulate the temporal intervals needed to cover all distances between each couple of waypoints.

Then, the geometric characteristics of the camera, also known as 'internal orientation of the camera', have to be found: the focal length, pixel dimensions and the frame format in order to correctly determine the physical area that is possible to acquire by each image. Estimated all these information and using a flight planning software, each waypoint is calculated in order to guarantee a minimum of $60 \%$ overlap between each couple of frames in the longitudinal direction, ensuring in this way also a stereoscopic vision and thus the threedimensionality of all objects in the model that will be recreated (Fig. 1). In addition to these parameters, another characteristic for the planning is the expected average scale of the photogram. The average scale of the photogram represents the relation between the dimension of an element in the photogram and the actual dimension of the element on the ground. Due to the fact that the terrain usually has not a flat morphology, the distance between each point of the image and the acquisition point is not the same, thus an average value of this distance is used instead to evaluate the average scale. The average scale is a guideline to correctly generate maps at the required scale. All this is undisputed for metric cameras on aerophotogrammetric flights; we want to investigate if this approach can be correct also for the multirotor UAVs using amateur or semimetric cameras [3]. 


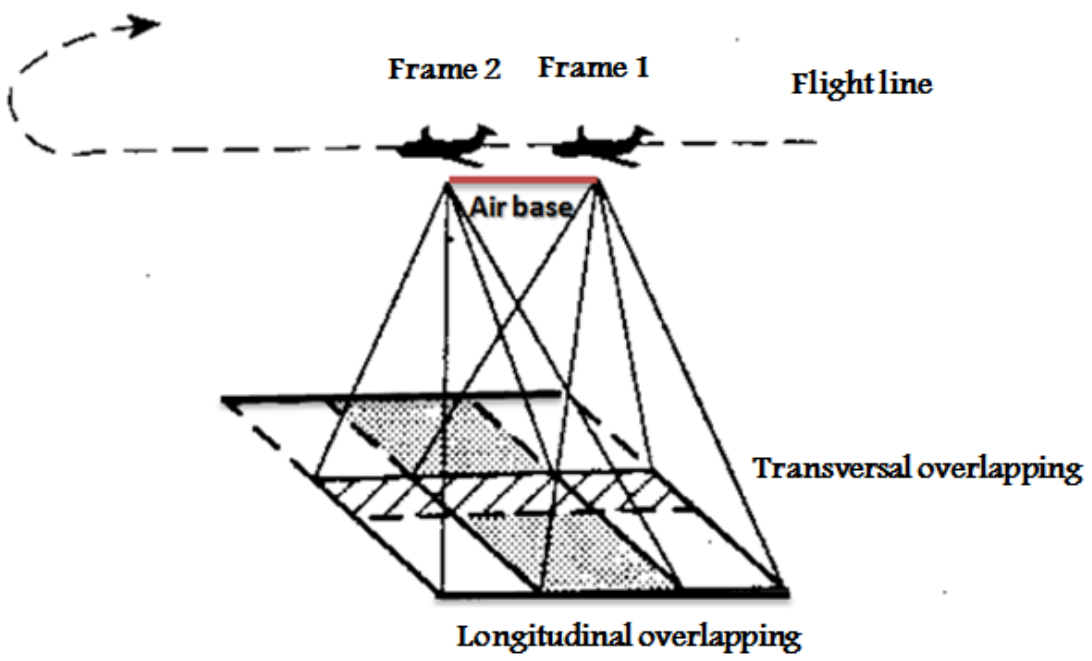

Figure 1: Air base, longitudinal overlapping and transversal overlapping in a flight planning (available online to http://www.grimessurveying.com).

The scope is to obtain all and only the frames needed to reconstruct the stereoscopy and to obtain geometrically correct reconstructions of the objects observed to reconstruct a DSMs [4]; for this reason an original piece of software ('UP23d': UAVs planner to 3 dimensional acquisitions) was developed to model the flight plan of the UAV for the acquisition optimization, evaluating at the same time if this approach is correct also for the multirotor UAVs using commercial or semi-metric cameras [5].

\section{Study area}

To realize this experiment a typical Italian square with historic-monumental buildings was chosen as field test. 'Piazza del Palazzo' is in the center of L'Aquila city and was deeply damage by the 2009 seismic main event [6]. Its dimensions are approximately 60 meters long to 38 meters wide and surrounded by the public library, the city hall 'Palazzo Margherita' with its $40 \mathrm{~m}$ height bell tower (Fig. 2).

This experimentation in the historic center of L'Aquila is a collaboration between the Faculties of Engineering of L'Aquila and 'La Sapienza' university of Rome with the technical support of IPT Company of Rome that gently provided a Mikrokopter platform in Okto configuration, including all on board sensors like a Reflex camera and GNSS navigation receivers. The choice of this platform was to achieve excellent flying characteristics even in presence of wind or other non optimal climatic conditions and according to the payload specified by the manufacturer. In fact a Canon EOS 550D equipped with a wireless video 


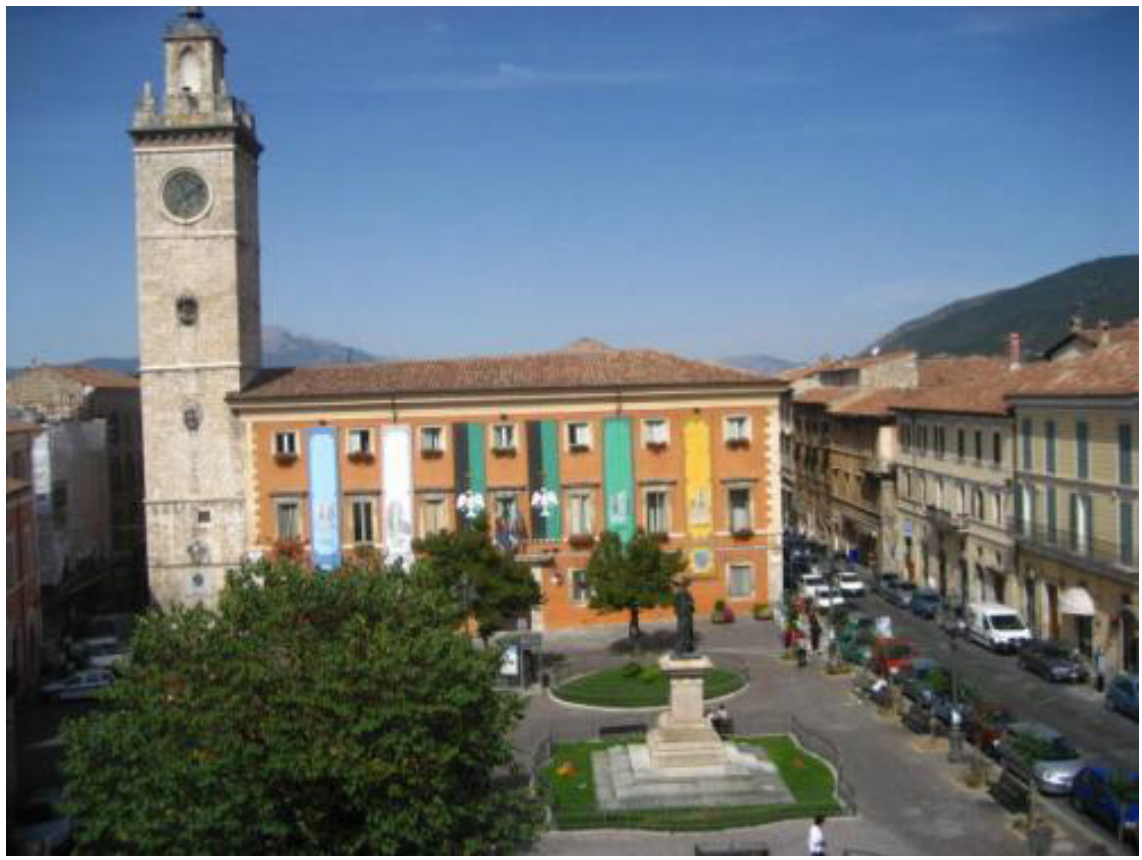

Figure 2: $\quad$ The test area: Piazza Palazzo.

transmission to the ground was chosen to allow a better control of the flight and acquisitions in real time.

After the UAV takeoff, the mode of autonomous flight can be activated; in such modality 15 frames all over the square were acquired during the tested 'manual' acquisition.

From this first analysis, we can note, that the number of acquisitions executed by the pilot in 'manual' modality was extremely redundant and this, as yet explained, is presently the most diffused way to acquire.

To simulate an acquisition based on our planning software we looked for two acquisitions that simulate those calculated by the software UP23d.

\section{Results}

The extraction of a DEM from a photogrammetric survey need at least a pair of frames, with a minimum overlap of $60 \%$, as the stereoscopy required to $3 \mathrm{D}$ survey is realized only in this area of overlap. In this experimentation, we chose two acquisitions between those made by the pilot UAVs that show geometric characteristics closest to those planned by the UP23d software. In the present case, the stereo pair frames a, were chosen with a distance between them of approximately $39 \mathrm{~m}$ and consequently with an overlap of $62 \%$.

To take advantage of stereoscopic vision for the extraction of DSM, it is necessary to reconstruct the geometry of the two images in relation to the 
surface, calculating position and orientation of the camera relative to the ground at the time of acquisition. The DEM obtained through the use of PCI Geomatics 2012 is shown in figure 3.

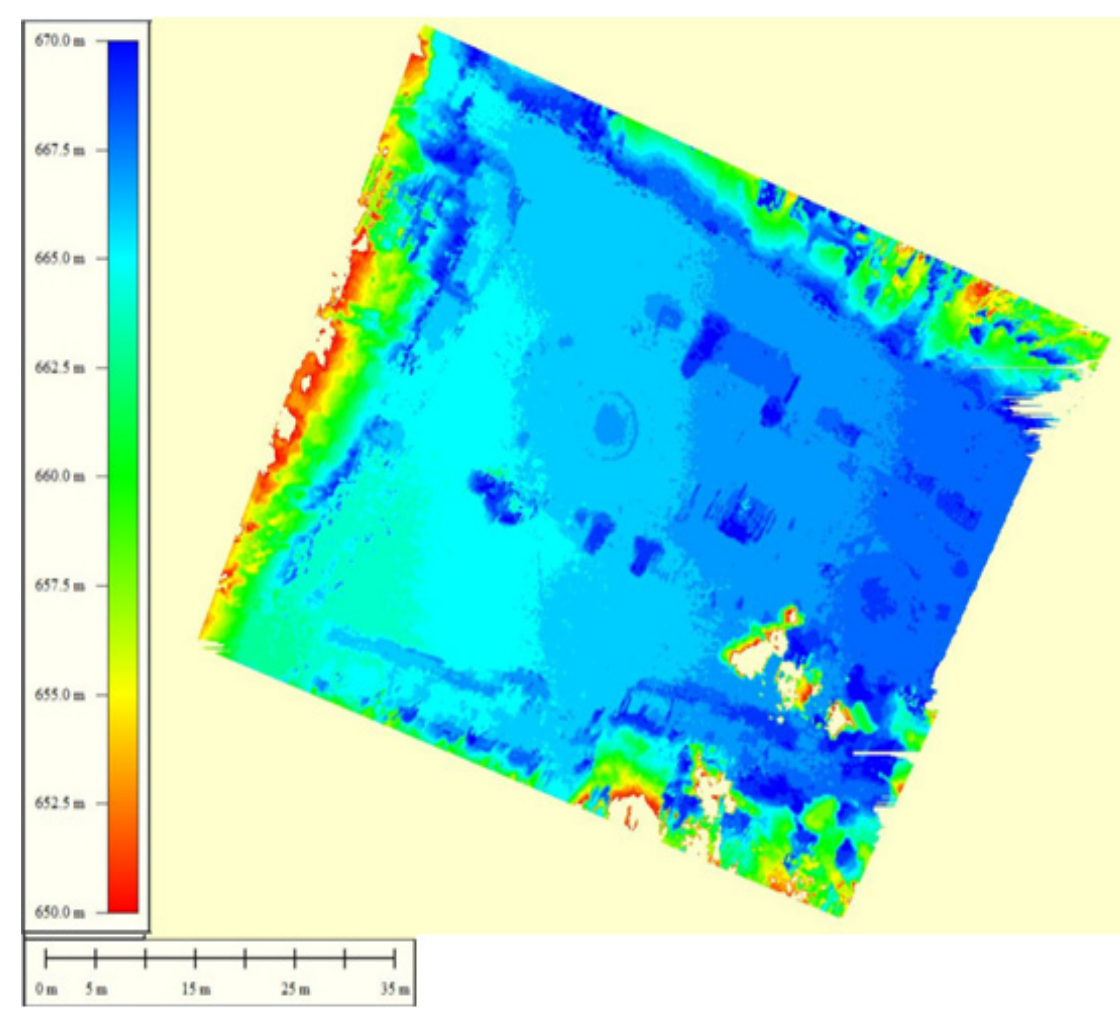

Figure 3: DSM resulting from the process of automatic extraction.

We can observe that an impressive precision characterizes the obtained DSM, in fact object with little elevation from the ground are visible: note the circular boardwalk in the centre of the image that is only 10 centimeter high from the ground. On the other hand, considering the accuracy only some very initial tests was possible to perform. Presently, in fact, only a small number of spot points extracted from medium-high scale digital cartography are available to check DSM elevations. From this introductory validation a maximum difference of around one meter was observed, and this value is contained in the expected error for the cartography at this scale.

This test only hints that the obtained DSM is more accurate than the cartography, but more has to be performed with a larger number of points with a higher accuracy: for this reason in this moment a laser scanning survey is in progress. So this technique seems to report an impressive precision and a very good accuracy, but it's still not evident if there are time and cost advantages 
comparing with more 'classical' methodologies; a weak point can be the need for a big number of GCPs, as an example in this test we used 21 differential GPS acquired points.

To evaluate the coherence of the obtained DSM with the actual objects present on the ground a high resolution orthophoto was realized showing another time a good fit (Fig. 4). It's important to underline that such precisions and accuracies are surely adequate to the early damage assessment in a post disaster scenario and in emergency management operations.

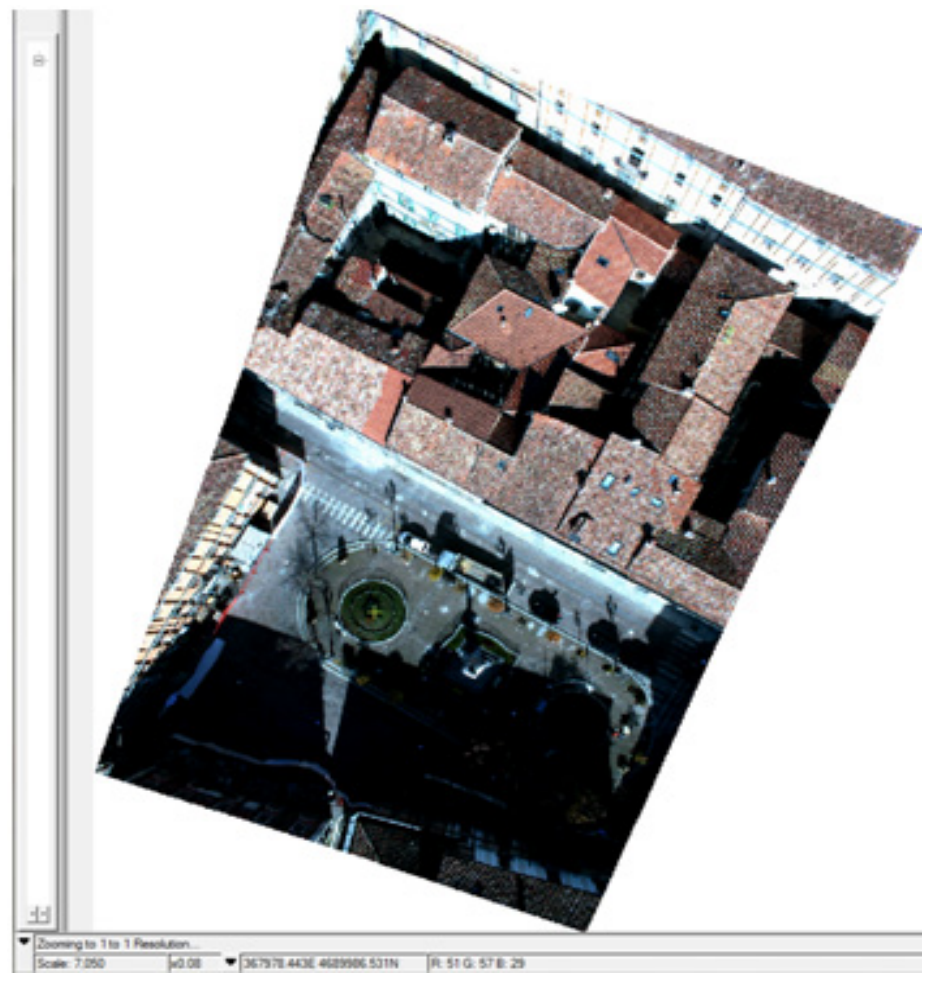

Figure 4: Orthophoto resulting from processing.

\section{Conclusion}

In this paper, we wanted to show the advantages of the use of UAVs in the case of post-earthquake survey scenarios, given the opportunity to fly, at low cost, near the objects we are interested in surveying and for reconnaissance operations also in inaccessible areas. In fact, the micro-mini UAV will have lower costs and more flexibility than any other survey instrument, producing a very detailed report of the damage. Also, in post disaster management operations, an UAV can survey dangerous parts of structures like the roofs that are inaccessible to human operators. In addition, it is not to be underestimated that, thanks to the electric 
engines, no gas emissions take place and virtually no noise is produced. In this work, some first results confirmed that the geometric conditions hypothesized are correct to automatically extract DSM but further and more extensive tests have to be performed. A laser scanning survey is in progress at the moment, and comparing it with the obtained results, it will be possible to have a correct evaluation of the accuracy.

\section{Acknowledgement}

The authors want to thank the IPT Company of Rome, for the availability of the Okto Mikrokopter platform, which made the test possible.

\section{References}

[1] Ioannis K. Nikolos, Kimon P. Valavanis, Senior Member, IEEE, Nikos C. Tsourveloudis, and Anargyros N. Kostaras. "Evolutionary Algorithm Based Offline/Online Path Planner for UAV Navigation" IEEE transactions on systems, man, and cybernetics - part b: cybernetics, vol. 33, no. 6, (2003).

[2] Karl Krau. 4th edition Italian translation Prof. Sergio DEQUAL Politecnico di Torino Cap 3. Pag. 147 - 153(1994).

[3] Ercolin L., Julitta F., Montagna M., Rigon P., Sarazzi D. "Moderne tecniche di rilievo aereo-fotogrammetrico di oggetti a sviluppo verticale per la produzione di DSM: il caso di Campione del Garda (BS)", ASITA 2011, (2011).

[4] F. Chiabrando, F. Nex, D. Piatti, F. Rinaudo. "UAV and RPV systems for photogrammetric surveys in archaeological areas: two tests in the Piedmont region (Italy)" Journal of Archaeological Science 38 697e710 (2011).

[5] V. Baiocchi, D. Dominici, M. Mormile. "UAV application in post - seismic environment" in proceedings of UAV-g 2013.

[6] V. Baiocchi, D. Dominici, M.V. Milone, M. Mormile. "Development of a software to plan UAVs stereoscopic flight: an application on post earthquake scenario in L'Aquila city" in proceedings of ICCSA 2013.

[7] Damien Eynard and Pascal Vasseur and Cedric Demonceaux and Vincent Fremont. "UAV Altitude Estimation by Mixed Stereoscopic Vision" The IEEE/RSJ International Conference on Intelligent Robots and Systems, 2010, Taipei, Taiwan (2010).

[8] Eisenbeiss H., Haarbrink R. B. "Accurate DSM production from unmanned helicopter systems" ISPRS Technical Commission VII Symposium 100 Years ISPRS Advancing Remote Sensing Science, Vienna, Austria (2010).

[9] John Tisdale, Zuwhan Kim, J. Karl Hedrick. "Autonomous UAV Path Planning and Estimation" IEEE Robotics and Automation Magazine, 10709932/09/2009 IEEE (2009).

[10] S. Hrabar. "3D Path Planning and Stereo-based Obstacle Avoidance for Rotorcraft UAVs" 2008 IEEE/RSJ International Conference on Intelligent Robots and Systems Acropolis Convention Center Nice, France, (2008). 